Pacific Journal of Mathematic 


\title{
CLASSES OF MINIMAL AND REPRESENTATIVE DOMAINS AND THEIR KERNEL FUNCTIONS
}

\author{
Michael MaschleR
}

1. Introduction. In connection with the problem of obtaining classes of conformally equivalent domains in the space of one or several complex variables, S. Bergman [3] introduced two kinds of canonical domains named minimal domains and representative domains. Since the mapping functions onto these domains were expressed in a closed form by using the Bergman kernel function and its derivatives, it was possible to deduce interesting properties of the kernel function which, in turn, provided more information about the canonical domains. (See S. Bergman [1] [3], M. Schiffer [9], M. Maschler [7]).

The object of this paper is to discuss "minimal domains" and "representative domains" with respect to certain subclasses of analytic functions, and to deduce solutions to some extremal problems. In addition, differential equations are obtained for the kernel function, which are valid for various classes of domains. The methods we use apply to the theory of functions of several complex variables as well, but first, the case of one complex variable should be clarified.

Let $D$ be a plane domain having a boundary of positive capacity. We consider the class of analytic functions $w=f(z)$ which have singlevalued, regular derivatives in $D$, and which possess developments of the form

$$
w=(z-t)+a_{m+1}(z-t)^{m+1}+a_{m+2}(z-t)^{m+2}+\cdots
$$

in the neighborhood of a point $t$ in $D$. There exists one function in this class which maps $D$ onto a domain having the smallest area ${ }^{1}$. This latter domain will be called an m-minimal domain with the origin as center. For $m=1$ we obtain the ordinary minimal domains.

As $w=f(z)$ may be multivalued and non-univalent, one has to extend the theory of the kernel function to domains on a Riemann surface, which may have "identified points", (That is, points which correspond to a single point of a univalent domain, under a conformal mapping). The ideas of this extension are not new and are treated here for the

Received January 7, 1959. This research was sponsored in part by the Office of Scientific Research of the Air Research and Development Command, United States Air Force, through its European Office, under Contract No. AF 61 (052)-04. Some of the results of this paper were presented in: (1) "Domain functions and conformal mappings with applications to extremal problems", a thesis for the Ph. D. degree at the Hebrew University, Jerusalem. (2) The Bull. of the Research council of Israel. Vol 7F (1957) p. 42. (Abstract).

1 The area is defined by $\iint_{D}\left|f^{\prime}(z)\right|^{2} d \omega$, where $d \omega$ is the area element. 
sake of completeness. We then treat Bergman's problem of minimizing the integral

$$
\iint_{D}|f(z)|^{2} d \omega
$$

where the functions $f(z)$ are single-valued, analytic, regular in the domain $D$ and satisfy, at a non-branch point $t$ in $D$, the condition

$$
f^{(\nu)}(t)=X_{\nu}, X_{\nu} \text { are constants, } \nu=0,1,2, \cdots, m .
$$

(See S. Bergman [2].) We prove that these minimizing functions are transformed, under a conformal mapping which is locally univalent at $t$, onto similar minimizing functions for the image domain, multiplied by the derivative of the mapping function. (The constants (1.3) are transformed linearly).

The mapping function onto an $m$-minimal domain can be expressed in a closed form in terms of the kernel function and its derivatives [Section 3]. This leads to a local condition for the kernel function, satisfied if and only if the kernel function belongs to an $m$-minimal domain.

Simply-connected $m$-minimal domains are always images of a 1 -minimal simply-connected domain (i. e., a circle), under a mapping function which is a polynomial of degree at most $m$, and vice versa [Section 4]. This is no longer true, in general, for the case of multiply-connected $\mathrm{m}$ minimal domains [Section 7]; however, each choice for the values of the first $m$ derivatives of the mapping functions at the center of a 1-minimal domain, determines a mapping onto an $m$-minimal domain with the same center [Section 4].

The shape of the doubly-connected 1-minimal domains is studied in Section 5. It is shown that the 1-minimal doubly-connected domain always has identified points, provided that no boundary component is reduced to a single point. Therefore, these minimal domains are different from those studied by $P$. Kufareff [6], which he obtained by restricting attention only to single-valued mapping functions.

Let $M_{D}(z, t)$ be a minimizing function of (1.2), for functions satisfying in (1.3) the values

$$
X_{0}=1, X_{1}=X_{2}=\cdots=\cdots X_{m-1}=0, \quad m \geqq 1 .
$$

Let $M_{D}^{*}(z, t)$ be a similar function for the case

$$
X_{0}=0, X_{1}=1, X_{2}=X_{3}=\cdots=X_{m}=0, \quad m \geqq 1 .
$$

The function $\left[M_{D}^{*}(z, t) / M_{D}(z, t)\right]$ satisfies (1.1) and remains invariant under a conformal mapping which satisfies (1.1) [Section 6]. This function is said to map $D$ onto an m-representative domain with the origin as center. In general, it is different from the $m$-minimal domain with the same center, but if both domains coincide and have the same center, 
say at the origin, then the minimizing functions for the $m$-minimal and $m$-representative domain $\Delta$ satisfy the differential equation

$$
\frac{d}{d \zeta}\left(\frac{M_{\Delta}^{*}(\zeta, 0)}{M_{\Delta}(\zeta, 0)}\right)=M_{\Delta}(\zeta, 0)
$$

for $\zeta \in \Delta$ [Section 6]. The interest in this relation is that it remains invariant under each transformation $w=f(\zeta)$ which satisfies $f^{\prime}(0)=1$, $f^{(\nu)}(0)=0, \nu=2,3, \cdots, m$. Thus, this relation holds for a general class of conformally equivalent domains.

2. The Bergman kernel function for generalized domains. Various extremal problems in conformal mappings yield, as solutions, a mapping function which may be meromorphic, and/or many-valued. In order to treat such problems, it is desirable to extend the concept of a domain and its Bergman kernel function. Making use of known ideas (see e.g., S. Bergman [4], p. 33, and R. Nevanlinna [8]), we proceed as follows: ${ }^{2}$

Let $D$ be a univalent domain in the $z$-plane, where boundary has a positive capacity. Let $w=w(z)$ be a function of $z$, defined for $z \in D$. We demand that $w^{\prime}(z)$ exists, that it is a single-valued, meromorphic function for $z \in D$, and does not vanish identically.

Among the set of points : $\{w(z) \mid z \in D\}$, we identify all the images of the same point $z . \quad w\left(z_{1}\right)$ and $w\left(z_{2}\right)$ are said to be different points if $z_{1} \neq z_{2}$. The obtained set is called : a generalized domain.

\section{EXAMPLES.}

a. If $D_{1}$ is the unit circle $|z|<1$, and $w(z)=z^{2}$, then the generalized domain $\Delta_{1}$ consists of two coverings of the unit circle with a branch point $w=0$.

b. If $D_{2}$ is the ring $1<|z|<e$, and $w(z)=\log z$, then the generalized domain $\Delta_{2}$ is the strip $0<\Re$ e $w<1$, where points $w_{1}$ and $w_{2}$, which satisfy

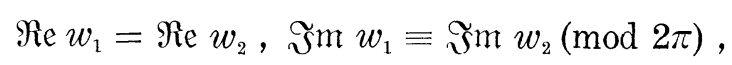

are identified.

DEFINITION. Let $\Delta$ be a generalized domain, obtained from a (classical) domain $D$, according to the above procedure. We say that a function $F(w)$ belongs to the class $\mathscr{L}^{2}(\Delta)$ if there exists a function $f(z)$, belonging to the $\operatorname{class}^{3} \mathscr{L}^{2}(D)$ such that

\footnotetext{
2 The proofs are simple and we omit them.
} 


$$
F(w)=f(z(w)) \cdot \frac{d z}{d w}, \quad z \in D
$$

It is clear that $F(w)$ may have a pole at a branch point of $\Delta$. Integration over the domain $\Delta$ is defined by the relation

$$
\iint_{\Lambda} G(w) d \omega=\iint_{D} G(w(z)) \cdot\left|w^{\prime}(z)\right|^{2} d \omega_{z},
$$

whenever the right-hand side exists. Here $d \omega_{z}$ denotes the area element in the $z$-plane.

Let $D$ be a univalent, positively bounded domain, without identified points, which generates, as described above, two generalized domains $\Delta$ and $\Delta^{*}$, then the mappings of $D$ onto $\Delta$ and $\Delta^{*}$ determine a one-to-one mapping :

$$
w^{*}=w^{*}(w), w \in \Delta,
$$

from $\Delta$ onto $\Delta^{*}$. This mapping will be called a conformal mapping. All these definitions do not depend on the particular choice of $D$.

From now on, unless otherwise stated, we shall use the term "domain" to mean a generalized domain. All such domains have the property that they can be mapped conformally onto univalent domains with a boundary of positive capacity and without identified points.

Let $D$ be a (generalized) domain. Introducing a scalar multiplication

$$
(f ; \bar{g})=\iint_{D} f \cdot \bar{g} d \omega,
$$

makes $\mathscr{L}^{2}(D)$ a Hilbert space which possesses a Bergman kernel function

$$
K_{D}(z, \bar{t})=\sum_{\nu=1}^{\infty} \rho_{\nu}(z) \overline{\varphi_{\nu}(t)},
$$

where $\varphi_{\nu}(z), \nu=1,2, \cdots$ is a complete orthonormal system.

The kernel function depends only on the domain $D$ and not on the particular choice of the complete orthonormal system. As a function of $z$, for each fixed $t$ which is not a branch point, the kernel function belongs to the class $\mathscr{L}^{2}(D)$. It may or may not be singular if $t$ is a branch point. $K_{D}(t, \bar{t})>0$ and takes the value infinity if $t$ is a branch point.

If a domain $D$ can be mapped conformally onto a domain $A$, by a mapping function $w(z), z \in D$, then the kernel functions of $D$ and $A$ satisfy the relation:

$$
K_{D}(z, \bar{t})=K_{\Delta}\left(w(z), \overline{w(t))} \cdot w^{\prime}(z) \cdot \overline{w^{\prime}(t)}, z, t \in D .\right.
$$

${ }^{3}$ A function $f(z)$ is said to belong to the class $\mathscr{L}^{2}(D)$ if it is single-values and regular in the domain $D$ and satisfies :

The integration is in the Lebesgue sense.

$$
\iint_{D}|f(z)|^{2} d x d y<\infty, z=x+i y
$$


(This relation is to be understood in the sense that the ratio between the two sides approaches 1 if $z$, or $t$, or both variables, approach a singularity point of the kernel function.) We shall end this section by stating an important theorem of S. Bergman for the generalized domains. (See [4], p. 26) :

THEOREm 1. Let $D$ be a (generalized) domain, and $t$ a fixed point in $D$, which is not a branch point. Consider the functions $f(z), z \in D$, which belong to the class $\mathscr{L}^{2}(D)$ and satisfy:

$$
f^{(\nu)}(t)=X_{\nu}, \nu=0,1,2, \cdots, m ;
$$

where $X_{\nu}$ are fixed complex numbers and $f^{(\nu)}(t)$ is the derivative of the order $\nu$ of $f(z)$ at the point $t$; then there exists among them one and only one function $M_{D}^{X_{0}},{ }^{x_{1}, \ldots,{ }_{m}(z, t)}$ which minimizes the integral $\iint_{D}|f(z)|^{2} d \omega$. This function can be represented in a closed form by using the kernel function and its derivatives:

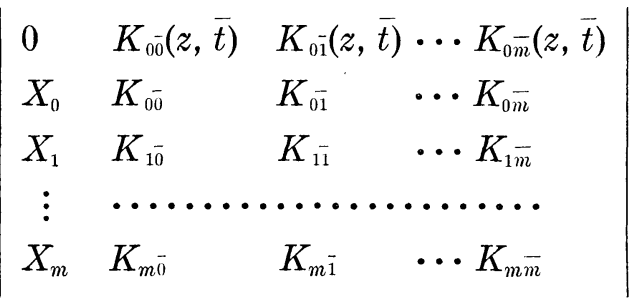

$$
\begin{aligned}
& M_{D}^{X^{0}},{ }^{X_{1}}, \ldots,{ }_{m}(z, t)=- \\
& K_{0 \overline{0}} \quad K_{\overline{01}} \cdots K_{0 \bar{m}} \\
& \begin{array}{llll}
K_{01} & K_{\overline{11}} & \cdots & K_{1 \bar{m}} \\
\cdots & \cdots & \cdots
\end{array}
\end{aligned}
$$

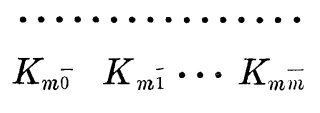

The value of the minimum is:

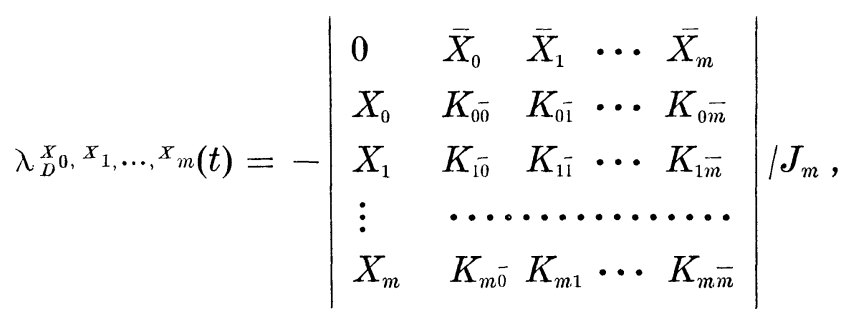

where $J_{m}$ denotes the denominator which appears in (2.9). 
Here

$$
\begin{array}{r}
K_{0 \bar{j}}(z, \bar{t})=\left[\frac{\partial^{j}}{\partial \zeta^{j}}\left(K_{D}(z, \bar{\zeta})\right]_{\bar{\zeta}=\bar{t}} \quad(j=0,1, \cdots, m) ;\right. \\
K_{i \bar{j}}=\left[\frac{\partial^{i+j}}{\partial z^{i} \partial \bar{\zeta}^{j}}\left(K_{D}(z, \bar{\zeta})\right)\right]_{z=t, \bar{\zeta}=\bar{i}}, \quad(i, j=0,1, \cdots, m) .
\end{array}
$$

( $i=0$, means that one should not differentiate with respect to $z$. Similarly, if $j=0$.)

Proof. This theorem was proved by S. Bergman for univalent, domains without identified points. ${ }^{4}$ ([4], pp. 26-27.) One can use the same proof, (which is based on the method of Lagrange multipliers), provided that one shows first that the minimum problem has a solution. This is done as follows: If $D$ is a generalized domain, then it can be obtained from a univalent, domain, without identified points $D^{*}$, by a conformal mapping $z=z\left(z^{*}\right), z^{*} \in D^{*}$. If $t^{*}$ is the inverse image of $t$, then

$$
\left.\frac{d z}{d z^{*}}\right|_{z^{*}=t^{*}} \neq 0, \infty
$$

because $t$ is not a branch point, and $D^{*}$ is univalent. Therefore, the inverse function $z^{*}=z^{*}(z)$ is regular at $t$ and $\left.\left(d z^{*} / d z\right)\right|_{z=t} \equiv c \neq 0$.

To each function $f(z)$ of the class $\mathscr{C}^{2}(D)$, corresponds one function $f^{*}\left(z^{*}\right)$ of the class $\mathscr{P}^{2}\left(D^{*}\right)$ such that

$$
f(z)=f^{*}\left(z^{*}(z)\right) \cdot \frac{d z^{*}}{d z},
$$

hence,

$$
\iint_{D}|f|^{2} d(1)=\iint_{D^{*}}\left|f^{*}\right|^{2} d(1)_{z^{*}}
$$

Thus, there is a one-to-one mapping between the family of functions considered in the theorem and the family of functions $f^{*}\left(z^{*}\right)$ of the class $\mathscr{L}^{2}\left(D^{*}\right)$ which satisfy :

$$
f^{*(\nu)}\left(t^{*}\right)=Y_{\nu}, \quad \nu=0,1,2, \cdots, m,
$$

where $Y_{\nu}$ are complex numbers satisfying the system of equations :

$$
\left[\frac{d^{\nu}}{d z^{*}}\left[f^{*}\left(z^{*}(z)\right) \frac{d z^{*}}{d z}\right]\right]_{z=t}=X_{\nu}, \quad \nu=0,1,2, \cdots, m .
$$

(See (2.8) and (2.14).) This system has one and only one solution because $c \neq 0$. Bergman's theorem ensures the existence of a unique func-

4 There always exist functions satisfying (2.8), if the boundary has a positive capacity. (See also K. I. Virtanen $\{\mathbf{1 1} \mid$.) 
tion which minimizes the right integral of (2.15) under the conditions (2.16) ; hence it follows from (2.14), (2.15) and (2.16) that this function multiplied by $d z^{*} / d z$ is the solution of the original problem, and that it is unique.

REMARK 1. Incidentally, we have proved that if $D$ and $D^{*}$ are two (generalized) domains, and $D^{*}$ is mapped conformally onto $D$ by the function $z=z\left(z^{*}\right), z^{*} \in D$, then, if $z^{*}=z^{*}(z)$ is the inverse mapping,

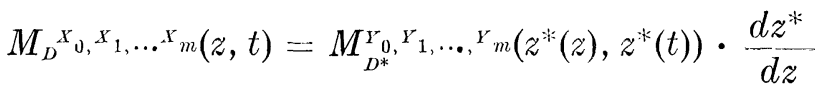

$$
\begin{aligned}
& \lambda_{D^{0}}^{X}{ }^{X_{1}}, \ldots,{ }^{X}{ }_{m}(t)=\lambda_{D *}^{Y_{0}}{ }^{Y_{1}}, \ldots,{ }^{Y} m\left(z^{*}(t)\right),
\end{aligned}
$$

where $Y_{\nu}, \nu=0,1,2, \cdots, m$, satisfy $(2.16),(2.17)$; provided that $t$ and $t^{*}=z^{*}(t)$ are not branch points of $D$ and $D^{*}$, respectively.

REMARK 2. It is possible to solve a similar extremal problem when $t$ is a branch point, but the solution depends on the type of the branch point at $t$. This solution will not be considered in this paper.

REMARK 3. If $t$ is not a branch point, then the denominator of the right-hand side of (2.9) is finite and positive.

Proof. Denote this denominator by $J_{m}$. It follows from (2.6) that $0<J_{0}=K_{0 \overline{0}}<\infty$, provided $t$ is not a branch point. Substituting $X_{0}=$ $X_{1}=\cdots=X_{m-1}=0, X_{m}=1$ in $(2.10)$, we obtain :

$$
\lambda_{D}^{0,0, \cdots, 0,1}(t)=\frac{J_{m-1}}{J_{m}}, \quad m \geqq 1 .
$$

But, by definition, and because of Theorem $1,0<\lambda_{i}^{n, 0, \cdots, 0,1}(t)<\infty$, hence, by induction, $0<J_{m}<\infty$.

3. Minimal domains with respect to almost identity mappings. In this section we shall be concerned with "almost identity" mappingfunctions, i. e., functions of the class $\mathscr{E}_{m, t}(D)$ defined as follows:

Definition. Let $D$ be a domain containing a point $t$ which is not a branch point. A function $f(z)$ is said to belong to the class $\mathscr{E}_{m, l}(D)$, if it satisfies the following conditions:

$$
f^{\prime}(z) \in \mathscr{L}^{2}(D) \quad \text { (see definition in Section 2), }
$$

$$
f(t)=0, f^{\prime}(t)=1, f^{\prime \prime}(t)=f^{(3)}(t)=\cdots=f^{(m)}(t)=0 ; \quad m \geqq 1 .
$$

This class has the following property: 
Lemma. If $\zeta=f(z)$ belongs to the class ' $\mathscr{C}_{m, t}(D)$ and maps $D$ onto $a$ domain $\Delta$, and if $w=\phi(\zeta)$ belongs to the class $\mathscr{E}_{m, 0}(\Delta)$, then $w=$ $W(z) \equiv \varphi(\zeta(z))$ belongs to the class $\mathscr{E}_{m, t}(D)$.

The proof is obvious.

The functions of the class $\mathscr{E}_{m, \iota}(D)$ map $D$ onto various domains, among which we look for that domain which has the least area ${ }^{5}$, and we try to determine the mapping function of $D$ onto this domain. It follows from the Lemma that the domain having the least area is an " $m$-minimal domain" with center at the origin, in the following sense:

Definition. A domain $\Delta$ is called an $m$-minimal domain having a center at a point $\tau$, if $\tau \in \Delta$ and is not a branch point of $\Delta$, and if any conformal mapping $w^{*}=w^{*}(w), w \in \Delta$, which satisfies $w^{*}(w) \in \mathscr{E}_{m, \tau}(\Delta)$, maps $\Delta$ onto a domain whose area is not smaller than the area of $\Delta$.

REMARK. It is clear that a translation $w^{*}=w+a, w \in \Delta$, maps an m-minimal domain with center at the point $\tau$ onto an m-minimal domain with center at the point $\tau+a$.

Denote by $M \equiv M_{D}(z, t)$, the function $M_{D}^{X_{0},{ }^{X_{1}}, \cdots, x_{m-1}}(z, t)$ (sec (2.9)), for the special case :

$$
X_{0}=1, X_{1}=X_{2}=\cdots=X_{m-1}=0,
$$

From Theorem 1 we obtain immediately:

THEOREM 2. Let $D$ be a (generalized) domain and $t$ a point in it, which is not a branch point, then there exists a unique function $f(z)$ satisfying the condition

$$
f(z) \in \mathscr{E}_{m, \iota}(D),
$$

which maps $D$ onto an m-minimal domain $\Delta$ with center at the origin. This mapping-function is given by

$$
f(t)=0, f^{\prime}(z)=M_{D}(z, t)
$$

(see (3.3)).

CoROLlary 1. If $D$ itself is an m-minimal domain with center at the point $t$, then $f(z)=z-t$ is the mapping-function required in Theorem 2. Therefore, in this case, $M_{D}(z, t)=1$. Hence, by (2.9) and (3.3), if $D$ is an m-minimal domain with center at the point $t$, then

5 The area of a domain $\Delta$ is defined as $\iint_{\Delta} d \omega$ (see (2.3)). Thus, different coverings are counted separately and, when identified points exist, only the fundamental domain is counted. 


$$
P_{m-1}(z, \bar{t})=\text { constant } \neq 0 .
$$

Here $P_{m-1}(z, \bar{t})$ denotes the domain function which is formed by crossing out the first and last coloumns and the second and last rows in the determinant which appears in the numerator of (2.9). $K=K_{D}$, (see (2.11), $(2.12))^{6}$.

CoRollary 2. The converse is also true. Indeed, if (3.6) is satisfied $^{5}$, then it follows from Theorem 2 that a mapping function of $D$ onto an m-minimal domain $\Delta$ with center at the origin is obtained by a translation $f(z)=z-t$; therefore, $D$ itself is an m-minimal domain with center at the point $t$.

The area $S_{\lrcorner}$of the $m$-minimal domain $d$ can be calculated from (2.10). Indeed, by (2.10),

$$
S_{\Delta}=Q_{m-1} / J_{m-1} .
$$

Here $Q_{m-1}$ is the determinant which is formed by crossing out the first, second and last rows and the first, second and last columns in the numerator of (2.10). $J_{m-1}$ was defined in Remark 3 of Section 2. (In the case $m=1$, we define $Q_{0}=1$ ).

TheOREM 3. A domain $D$ containing a point $t$, which is not a branch point, is an m-minimal domain with center at the point $t$, if and only if the right-hand side of (3.7) is equal to the area $S_{D}$ of $D$.

Proof. By Theorem 2, $D$ can be mapped onto an $m$-minimal domain $\Delta$ having an area given by (3.7). If $S_{\Delta}=S_{D}, D$ itself is an $m$-minimal domain. If $D$ is an $m$-minimal domain then $S_{4}=S_{D}$.

REMARK. Observe that the right-hand side of (3.7) depends only on the kernel function and its derivatives at the single point $t$.

1-minimal domains were introduced by S. Bergman and their definition was later extended to domains in the space of $n$-complex variables. Some properties of 1-minimal domains were studied by S. Bergman [3] [4], by M. Schiffer [9] and by the present author [7]. We shall see that many properties of 1-minimal domains can be extended to properties of $m$-minimal domains, and that these new properties yield information about the behaviour of the kernel function as well as distortion theorems for certain classes of domains.

4. Simply-connected $m$-minimal domains. It is known that a simplyconnected 1-minimal domain can only be a circle, the center of which is

${ }_{6}$ The constant on the right-hand side of (3.6) cannot be zero, because $M_{D}(t, t)=1$ and the denominator of the right-hand side of (2.9) is finite and positive (see Remark 3 of Section 2). 
the center in the sense of the definition of a minimal domain. We shall show, in this section, that the class of all simply-connected $m$-minimal domains can be obtained from a circle by mapping-functions which are polynomials of degree (at most) $m$. First, we consider the mapping of any domain $D$ (not necessarily simply-connected) onto an $m$-minimal domain.

THEOREM 4. Let $D$ be an m-minimal domain ( $m \geqq 1$ ), having a center at the origin. Let $D^{*}$ be a domain, containing the origin and being locally univalent there, which can be mapped conformally onto $D$ by a transformation $z=z\left(z^{*}\right), z^{*} \in D^{*}$, which satisfies $z(0)=0$; then

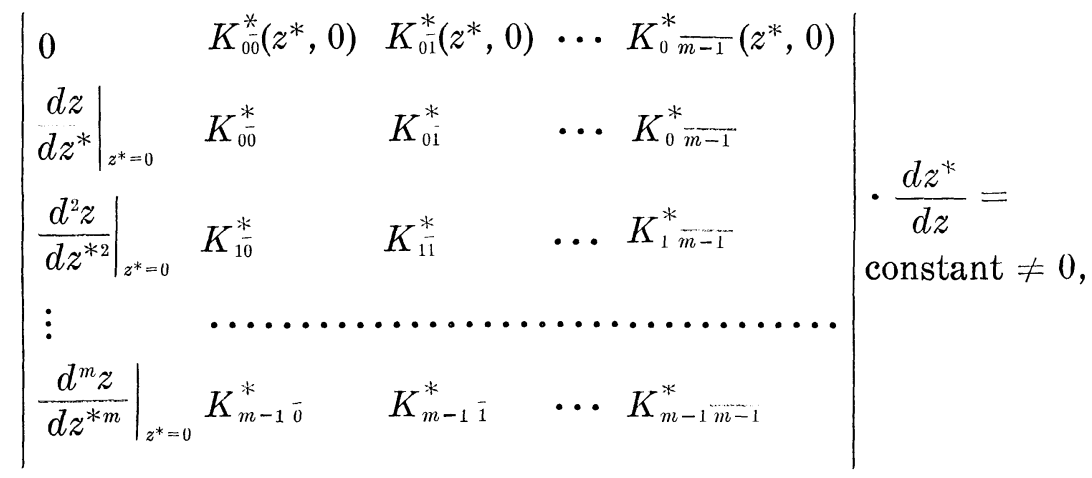

$z^{*} \in D^{*}$. Here $K^{*}\left(z^{*}, \bar{\zeta}^{*}\right) \equiv K_{D^{*}}\left(z^{*}, \bar{\zeta}^{*}\right) ; d z /\left.d z^{*}\right|_{z^{*}=0} \neq 0 ; t^{*}=0$. (See (2.11)(2.12).) Conversely: if (4.1) is satisfied, and $d z / d z^{*} \neq 0$, then $z=z\left(z^{*}\right)$ maps $D^{*}$ onto an m-minimal domain.

Proof. If $D$ is an $m$-minimal domain with center at the origin, then $M_{D}(z, 0)=$ constant. $\quad(\text { See Section } 3 \text {, Corollary 1. })^{7}$ Choosing $f^{*}\left(z^{*}\right)=d z / d z^{*}$ and substituting it in (2.16), one observes that (2.17) is satisfied (since $\left.X_{0}=1, X_{1}=X_{2}=\cdots=X_{m-1}=0\right)$. Therefore, by (2.18),

$$
M_{D}(z, 0)=M_{D *}^{Y_{0}{ }^{Y_{1}}, \ldots,{ }^{Y},{ }_{m-1}\left(z^{*}(z), 0\right)} \cdot \frac{d z^{*}}{d z},
$$

where

$$
Y_{\nu}=\left.\frac{d^{\nu+1} z}{d z^{* \nu+1}}\right|_{z^{*}=0}, \nu=0,1, \cdots, m-1
$$

Thus the relation (4.1) has been established. The converse statement is obtained by reversing the order of these arguments, and by using Corollary 2 of Section 3.

If $D$ is a simply-connected $m$-minimal domain, we can assume that $D^{*}$ is the unit circle. Since the kernel function of the unit circle is

7 For the definition of $M_{D}(z, 0)$, see (3.3). 


$$
K_{D^{*}}\left(z^{*}, \bar{\zeta}^{*}\right)=\frac{1}{\pi} \frac{1}{\left(1-z^{*} \bar{\zeta}^{*}\right)^{2}},
$$

(see [4] p. 9), it follows that

$$
\begin{aligned}
& K_{0 j}^{*}\left(z^{*}, 0\right)=\frac{1}{\pi}(j+1) ! z^{* j}, \\
& \pi K_{i j}^{*}=\left\{\begin{array}{l}
0 \quad, i \neq j \\
(j+1) ! j !, i=j
\end{array} \quad, i, j=0,1,2, \cdots .\right.
\end{aligned}
$$

Let $z=z\left(z^{*}\right)=a_{1} z^{*}+\left(a_{2} z^{* 2}\right) / 2 !+\left(a_{3} z^{* 3}\right) / 3 !+\cdots$ in the neighbourhood of the origin, $a_{1} \neq 0$; then, substitution of (4.4) and (4.5) in (4.1) yields, after some trivial calculations,

$$
a_{1}+\frac{a_{2}}{1 !} z^{*}+\frac{a_{3}}{2 !} z^{*^{2}}+\cdots+\frac{a_{m}}{(m-1) !} z^{* m-1}=C \cdot \frac{d z}{d z^{*}} .
$$

Therefore, the constant $C$ is equal to 1 and all the derivatives of $z\left(z^{*}\right)$ of an order greater than $m$ vanish at the origin. Thus we have proved:

TheOREM 5. Any polynomial of degree $m$ having a non-zero derivative at the origin maps the circle about the origin onto an m-minimal domain whose center is the image of the center of the circle. And conversely, any simply-connected m-minimal domain can be obtained from a circle by a mapping whose function is a polynomial of degree (at most) $m$, the derivative of which is not zero at the origin.

Theorem 5 suggests that perhaps all $m$-minimal multiply-connected domains are images of 1-minimal domains under polynomial mappings. This however, is not true in general, as we shall see later (see Section 7). Nevertheless, each $p$-connected 1-minimal domain generates a class of $p$-connected $m$-minimal domains conformally equivalent to it; this class has $m+1$ complex degrees of freedom. Indeed, since any domain can be mapped onto a 1-minimal domain such that a non-branch fixed point corresponds to its center, we can assume that the domain $D^{*}$ of Theorem 4 is a 1-minimal domain having a center at the origin. A necessary and sufficient condition for $D^{*}$ to be such a domain is : $K_{D^{*}}\left(z^{*}, 0\right)=$ constant $\neq 0$ (see (3.7); see also [7]); therefore, $K_{i \overline{0}}^{*_{\overline{0}}}=K_{0 \bar{i}}^{*}=0, i=1,2,3, \cdots$, and (4.1) reduces to:

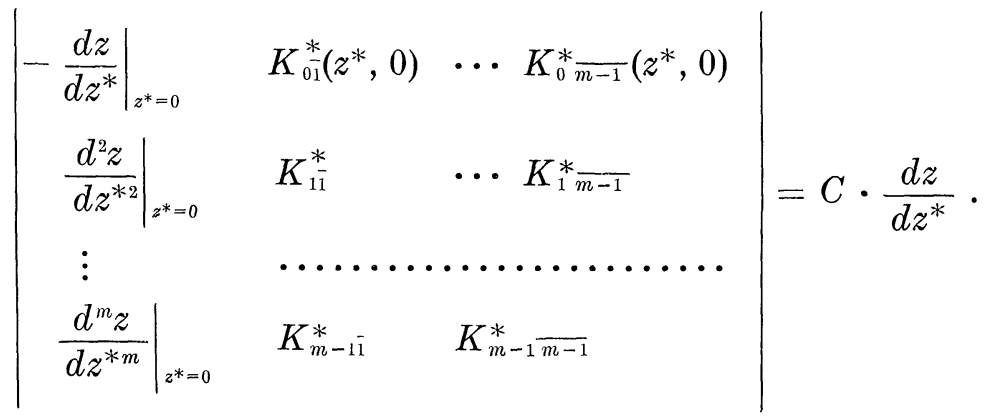


Here, $K^{*}\left(z, \bar{\zeta}^{*}\right)=K_{D^{*}}\left(z^{*}, \bar{\zeta}^{*}\right), d z /\left.d z^{*}\right|_{z^{*}=0} \neq 0$ and $C=$ constant $\neq 0$ must be equal to minus the minor of the element $-(d z) /\left.d z^{*}\right|_{z^{*}=0}$. Thus, the conformal mapping $z=z\left(z^{*}\right)$ maps the 1-minimal domain $D^{*}$ with center at the origin onto an $m$-minimal domain, $m \geqq 1$ such that the centers correspond, if and only if $z=z\left(z^{*}\right)$ satisfies (4.7).

Let us choose arbitrary constants $-c_{1}, c_{2}, c_{3}, \cdots, c_{m}, c_{1} \neq 0$, for the elements of the first column of the determinant in $(4.7)$; then $d z / d z^{*}$, thus defined, will indeed satisfy $\left(d^{k} z\right) /\left.d z^{* k}\right|_{z^{*}=0}=c_{k}^{8}$.

Since, moreover, a translation carries an $m$-minimal domain onto an $m$-minimal domain, we have arrived at

TheOREM 6. Let $D$ be a 1-minimal domain having a center at the origin; then, to each choice of $m+1$ constants, $c_{0}, c_{1}, c_{2}, c_{3}, c_{m}, c_{1} \neq 0$. there exists one and only one function.

$$
z=c_{0}+c_{1} z^{*}+\frac{1}{2 !} c_{2} z^{*^{2}}+\cdots+\frac{1}{m !} c_{m} z^{*^{m}}+d_{m+1} z^{* m+1}+d_{m+2} z^{* m+2}+\cdots,
$$

which maps $D^{*}$ onto an m-minimal domain with center at the point $c_{0}$. The mapping function is given by (4.7) where $\left(d^{k} z\right) /\left.d z^{* h}\right|_{z^{*}=0}$ are replaced by $c_{k}$.

Corollary. In general, $d_{j}, j=m+1, m+2, \cdots$, depend on the choice of $c_{2}, c_{3} \cdots, c_{m}$, but they do not depend on the choice of $c_{0}$ and $c_{1}$. Indeed, in order to obtain $d_{j}$, one has to differentiate $j$ times the lefthand side of (4.7) and to put $z^{*}=0$. The resulting expression does not contain either $c_{0}$ or $c_{1}$.

5. Doubly-connected 1-minimal domains. There is an unpublished result of $M$. Schiffer stating that univalent finitely-connected domains which do not possess identified points cannot be 1-minimal domains unless they are circles punctured at isolated points (the center of the circles is not punctured).

P. Kuffareff [6] studied the normalized conformal mapping of a ring onto a domain having a minimal area, restricting the mapping-function to be single-valued, and he found out that the minimal domain thus obtained lies on a double-sheeted Riemann surface. It seems natural to ask whether the use of a wider class of mapping-functions, i. e. integrals of functions of the class $\mathscr{L}^{2}$, yields different minimal domains. We shall show that this is indeed the case : a 1-minimal domain which is conformally equivalent to the ring always possesses identified points; hence, the mapping-function from the ring onto it is multi-valued; in

8 This is shown by differentiating (4.7) $l \varepsilon$ times and putting $z^{*}=0$. 
some cases the minimal domain is even a univalent domain (with identified points).

Let $T$ be the ring $0<r<|z|<1$ in the $z$-plane. It is known (see $[4]$, p. 10) that its kernel function is

$$
K_{T}(z, \bar{t})=\frac{1}{\pi z \bar{t}}\left[\mathfrak{p}\{\log (z \bar{t})\}+\frac{\eta_{1}}{\omega_{1}}\right] .
$$

Here $p(v)$ is the Weierstrass elliptic function having the periods $2 \omega_{1}=-2 \log r, 2 \omega_{3}=2 \pi i, \eta_{1}=\zeta\left(\omega_{1}\right)$, where $\zeta(v)$ is the corresponding Weierstrass zeta-function. Let $t$ be a fixed real point in the ring $T$, then the mapping

$$
u=\log z+\log t-2 \log r
$$

will map the ring onto a domain $S$ which is an infinite strip

$$
\log t-\log r<\Re e \quad u<\log t-2 \log r,
$$

in which the points $u \pm 2 k \pi i, k=0,1,2, \cdots$ are identified. Let $A B C D$ be the fundamental rectangle of $S: A \equiv \log t-\log r, B \equiv \log t-2 \log r$, $A, B$ are real ; $C \equiv B+2 \pi i, D \equiv A+2 \pi i$. It follows from (2.7) that the kernel function of $S$ satisfies:

$$
K_{S}(u, \bar{\tau})=\frac{1}{\pi}\left[\mathfrak{p}(u)+\frac{\eta_{1}}{\omega_{1}}\right], \quad \tau=2 \log t-2 \log r .
$$

Our aim is to map $T$ onto a 1-minimal domain in such a way that the point $t$ will correspond to its center. (From symmetry considerations it follows that the generality of the mapping is not affected by the fact that $t$ is required to be real). In order to achieve this, we first map $T$ onto $S$ and then map $S$ onto a 1-minimal domain ( $\tau$ corresponds to its center). This last mapping is produced by the function

$$
w=-\pi \int_{\tau}^{u} K_{S}(v, \bar{\tau}) d v+\zeta(\tau)-\frac{\eta_{1}}{\omega_{1}} \tau=\zeta(u)-\frac{\eta_{1}}{\omega_{1}} u
$$

(see Theorem 2, $(2.9) ; m=1$ ).

(All other 1-minimal domains whose centers correspond to $t$ are obtained from this one by the mapping $W=c_{0}+c_{1}\left(w-\zeta(\tau)+\left(\eta_{1} / \omega_{1}\right) \tau, c_{1} \neq 0\right.$; see Corollary in Section 4).

Let $\Delta$ be the minimal domain obtained from $S$ by the mapping (5.5). It follows from the quasi-periodicity of the zeta function that

$$
w(u \pm 2 k \pi i)-w(u)= \pm k \frac{\pi i}{\log r}, \quad k=0,1,2, \cdots ;
$$

hence, the points $w \pm k \pi i / \log r$ are identified in $\Delta$. 
It remains to find the fundamental domain of $\Delta$ which is the image of the rectangle $A B C D$ under the mapping (5.5). For this purpose we first determine the image under the same mapping of the rectangle $E F G H$, where $E \equiv 0, F \equiv \omega_{1}=-\log r G=-\omega_{2}=-\log r+\pi i H \equiv \omega_{3}=\pi i$ together with its reflections $F E^{\prime} H^{\prime} G\left(E^{\prime}=2 \omega_{1}, H^{\prime}=2 \omega_{1}+\pi i\right), H G F^{\prime \prime} E^{\prime \prime}$ $\left(F^{\prime \prime}=\omega_{1}+2 \pi i, E^{\prime \prime}=2 \pi i\right)$ and $G H^{\prime} E^{\prime \prime \prime} F^{\prime \prime}\left(E^{\prime \prime \prime}=2 \omega_{1}+2 \pi i\right)$. (See Figure 1.)

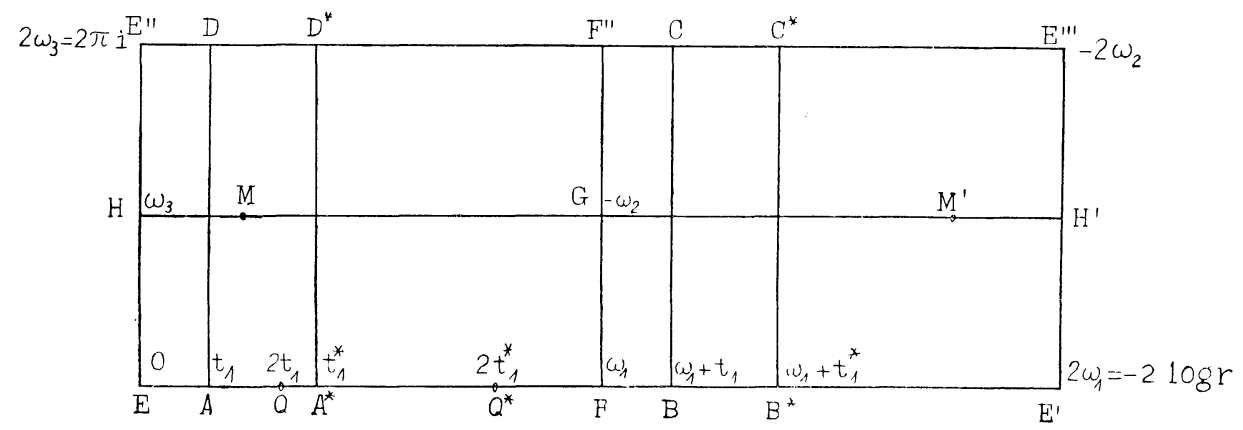

Figure 1. $u$-plane $t_{1}=\log t-\log r$

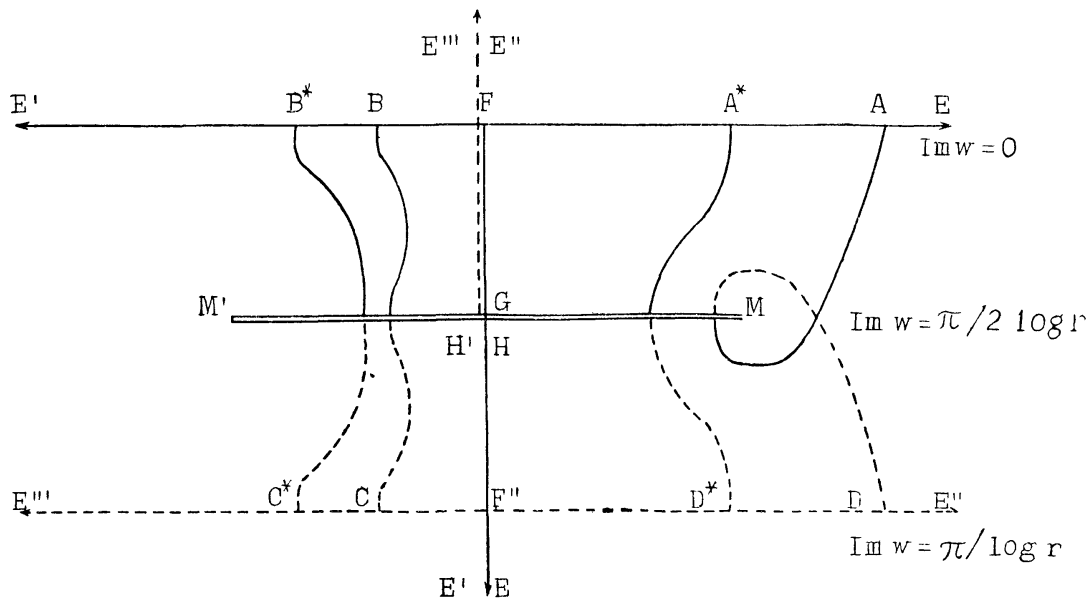

Figure 2. $w$-plane

The image can be deduced from [5] p. 190 : there are two and only two points, $M$ on $H G$ and its symmetric point $M^{\prime}$ on $G H^{\prime}$, where $-w^{\prime}(u)=\mathfrak{p}(u)+\eta_{1} / \omega_{1}=0$. These points correspond to the two branch points of the first order in the image which are on the line ₹mi $w=$ $(\pi) / 2 \log r$. The image of the four rectangles lies on a two-sheeted Riemann surface as shown in Figure 2. (We denote points in the $w$-plane which correspond to points in the $u$-plane by the same letter. Lines on one sheet are traced by a dotted line.)

In order to obtain more information about the image of the rectangle $A B C D$, we shall prove : 
LEMma. The distance $\overline{M H}$ in the u-plane is smaller than the distance $\overline{M G}$.

Proof. $\mathfrak{p}(u)+\eta_{1} / \omega_{1}$ is real and a monotone function and it takes all the values from $-\infty$ to $+\infty$ as $u$ traces the line EHGFE. (The function $\mathfrak{p}(u)$ has these properties and (see [10] p. 184)

$$
\eta_{1}=\frac{\pi^{2}}{2 \omega_{1}}\left[1 / 6+\sum_{n=1}^{\infty} 1 / \sin ^{2} \pi \frac{n \omega_{3}}{\omega_{1}}\right]
$$

is real because each term is real; so also is $\left.\omega_{1}\right)$. This function vanishes at the point $M$ on $H G$; therefore, it is negative at the point $H$. It remains to show that

$$
\mathfrak{p}\left(\omega_{3}+\frac{1}{2} \omega_{1}\right)+\frac{\eta_{1}}{\omega_{1}}>0
$$

Indeed,

$$
\mathfrak{p}(u)+\frac{\eta_{1}}{\omega_{1}}=\frac{\pi^{2}}{4 \omega_{1}^{2}} \cdot \sum_{n=-\infty}^{\infty} 1 / \sin ^{2}\left(\pi \frac{u-2 n \omega_{3}}{2 \omega_{1}}\right)
$$

(see [10] p. 184); it is real for $u=\omega_{3}+\frac{1}{2} \omega_{1}$, hence it is sufficient to consider the real part of each term of the series. It is easy to verify that, for $u=\omega_{3}+\frac{1}{2} \omega_{1}$, the real part of each term of the right-hand side of (5.7) is positive; hence $M$ lies between $\omega_{3}$ and $\omega_{3}+\frac{1}{2} \omega_{1}$.

From this lemma it follows that three possibilities can occur (taking various values for $t$ ):

(i) The rectangle $A B C D$ in the u-plane contains $M$ but does not contain $M^{\prime}$.

(ii) This rectangle, which we now denote by $A^{*} B^{*} C^{*} D^{*}$, contains neither $M$ nor $M^{\prime}$.

(iii) This rectangle, which we now denote by $A^{* *} B^{* *} C^{* *} D^{* *}$, contains $M^{\prime}$ but does not contain $M$.

In the first and third case, the minimal domain $\Delta$ will contain one branch point and will thus lie on a two-sheeted Riemann surface (and it will, of course, possess identified points). In the second case, only one sheet is required for the minimal domain (which, however, still possesses identified points).

The figure shows only the fundamental domain (for the cases (i) and (ii)). The images of the lines $A D, B C, A^{*} D^{*}, B^{*} C^{*}$ are not exact. The center of the minimal domain lies on the real axis of the $w$-plane and it is the image of the point $u=2 \log t-2 \log r$ (see 5.4).

6. m-representative domains. Attempts to generalize the Riemann mapping theorem to the case of domains in the $n$ complex dimensional space lead to various other classes of canonical domains. A well known 
class is the class of the "representative domains, introduced by S. Bergman (see e. g. [1] [3]).

In this section we shall limit ourselves to the case of a plane domain and generalize the concept of the representative domains so that the mapping functions onto these new canonical domains will satisfy the relations (3.2).

Definition. Let $M_{D}^{*}(z, t)$ be equal to $M_{D}^{X^{0}},{ }^{x_{1}}, \ldots x_{m}(z, t)$ (see (2.9)), for

$$
X_{0}=0, X_{1}=1, X_{2}=X_{3}=\cdots=X_{m}=0
$$

Let $M_{D}(z, t)$ be defined again by (3.3). Here $t \in D$ and is not a branch point. The function

$$
f(z)=\frac{M_{D}^{\prime}(z, t)}{M_{D}(z, t)} ; m \geqq 1
$$

satisfies the relation

$$
f(t)=0, f^{\prime}(t)=1, f^{\nu}(t)=0 ; 2 \leqq \nu \leqq m,
$$

and maps $D$ onto a domain $\Delta$. The domain $\Delta$ will be called an $m$-representative domain with center at the origin. An $m$-representative domain with a different center is obtained by a translation.

These $m$-representative domains are indeed canonical domains in the sense of the following.

THEOREM 7. If a domain $D$ in the z-plane is mapped onto a domain $D^{*}$ in the $\zeta$-plane by a function $\zeta=\zeta(z)$ which satisfies

$$
\zeta(t)=0, \zeta^{\prime}(t)=1, \zeta^{(\nu)}(t)=0 ; 2 \leqq \nu \leqq m,
$$

and $t$ is a non-branch point in $D$, then

$$
\frac{M_{D}^{*}(z, t)}{M_{D}(z, t)}=\frac{M_{D^{*}}^{*}(\zeta(z), 0)}{M_{D^{*}}(\zeta(z), 0)}
$$

Thus $D$ and $D^{*}$ generate the same m-representative domain.

Proof. Replacing $m$ by $m-1$ and $z$ by $\zeta$ in (2.16) and (2.17), we see that

$$
\begin{aligned}
& X_{0}=1, X_{1}=X_{2}=\cdots=X_{m-1}=0 \\
& Y_{0}=1, Y_{1}=Y_{2}=\cdots=Y_{m-1}=0
\end{aligned}
$$

satisfy the equations (2.16) and (2.17); therefore 


$$
M_{D}(z, t)=M_{D^{*}}(\zeta(z), 0) \cdot \frac{d \zeta}{d z}
$$

(See Remark 1 in Section 2.) Similarly,

$$
M_{D}^{*}(z, t)=M_{D^{*}}^{*}(\zeta(z), 0) \frac{d \zeta}{d z} .
$$

The relation (6.5) now follows from (6.8) and (6.9).

In general, $m$-minimal domains are different from $m$-representative domains. (See Section 7.) It is, therefore, interesting to look for properties of domains which are simultanuously $m$-minimal and $m$-representative, with the same center.

THEOREM 8. If $D$ is an m-minimal domain and also an m-representative domain, with the same center $t$ then

$$
M_{D}^{*}(z, t)=z-t
$$

Proof. On the one hand $D$ is an $m$-minimal domain, therefore the mapping

$$
w=f(z)=z-t
$$

maps it onto an $m$-minimal domain $\Delta$ with the origin as center; hence it is implied from (3.5) that

$$
M_{D}(z, t)=1, \quad z \in D .
$$

On the other hand, $\Delta$ is also an $m$-representative domain with a center at the origin, hence (6.2) and (6.12) imply

$$
w=M_{D}^{*}(w, 0) ;
$$

therefore, by (6.9) we obtain the relation (6.10).

By reversing the arguments of the proof we obtain immediately the converse theorem:

THEOREM 9. If a domain $D$ is an m-minimal domain with $t$ as center, and its kernel function with its derivatives satisfy the relation (6.10), then $D$ is also an m-representative domain with the same center.

Proof. It follows from (6.12) (6.2), and (6.10) that $w=z-t$ maps $D$ onto an $m$-representative domain with the origin as center, therefore, $D$ itself is an $m$-representative domain with $t$ as center.

Using the transformation formulas for $M_{D}(z, t)$ and $M_{D}^{*}(z, t)$, under conformal mappings, one can now obtain a differential equation for the kernel functions of the class of all domains which are obtained from the 
domain $D$ of the previous theorem by a mapping satisfying (6.4).

Theorem 10. For each domain $D^{*}$ which is conformally equivalent to the domain $D$ of Theorem 8, and for which the mapping function $\zeta=\zeta(z)$ satisfies (6.4), there exists a differential equation for $K_{D^{*}}(\zeta, \bar{\tau})$. This equation can be put in the form

$$
\frac{d}{d \zeta}\left(\frac{M_{D^{*}}^{*}(\zeta, 0)}{M_{D^{*}}(\zeta, 0)}\right)=M_{D^{*}}(\zeta, 0)
$$

Proof. Formulas (6.5), (6.10), (6.12) imply

$$
z-t=M_{D^{*}}^{*}(\zeta, 0) / M_{I * *}(\zeta, 0) .
$$

(6.8) and (6.12) imply

$$
\frac{d z}{d \zeta}=M_{D^{*}}(\zeta, 0)
$$

Equation (6.14) is obtained now by differentiating (6.15) with respect to $\zeta$.

REMARK. For the case $m=1$, one has

$$
\begin{gathered}
M_{D^{*}}^{*}(\zeta, 0)=-\frac{K_{01}^{*} K_{01}^{*_{-}}(\zeta, 0)-K_{00}^{*_{-}} K_{01}^{*_{1}}(\zeta, 0)}{K_{00}^{*_{-}} K_{1 \overline{1}}^{*_{\overline{1}}}-K_{01}^{*_{-}} K_{10}^{*_{0}}}, \\
M_{D^{*}}(\zeta, 0)=K_{00}^{*_{-}}(\zeta, 0) / K_{00}^{*_{-}} ;
\end{gathered}
$$

where $K^{*}=K_{D^{*}}$. Inserting this in (6.14), one obtains, after some calculations the relation

$$
\frac{1}{\left[K_{D^{*}}(\zeta, 0)\right]^{3}}\left|\begin{array}{cc}
K_{D^{*}}(\zeta, 0) & \frac{\partial K_{D^{*}}(\zeta, 0)}{\partial \zeta} \\
\frac{\partial K_{D}^{*}(\zeta, 0)}{\partial \bar{\tau}} & \frac{\partial^{2} K_{D^{*}}(\zeta, 0)}{\partial \zeta \partial \bar{\tau}}
\end{array}\right|=\text { const. }
$$

This relation and its generalization to the case of domains in the $n$ complex dimensional space was proved in [7].

7. A counter example. It is interesting to note that Theorem 5 no longer holds, in general, if we replace the circle by a 1-minimal multiply-connected domain $D$. A counter-example is an obvious deduction from the following theorem. 
Theorem 11. If a 1-minimal domain D, with the origin as center, is mapped onto a 2-minimal domain with the origin as center, and the mapping function is a polynomial

$$
w=\alpha_{1} z+\frac{1}{2} a_{2} z^{2}, a_{1}, a_{2} \neq 0,
$$

then $D$ is also a 1-representative domain with the origin as center.

Proof. $D$ is a 1-minimal domain with the origin as center; therefore, $K(z, 0) \equiv K_{l}(z, 0)=$ constant, for $z \in D$. (See Corollary 1, Section 3.) This implies that $K_{10}=K_{0 \overline{1}}=0$. Hence, it follows from Theorem 4 that

$$
-a_{1} K_{00} K_{11}-a_{2} K_{01}(z, 0) K_{0 \overline{0}}=c \cdot\left(a_{1}+a_{2} z\right) .
$$

Thus, the value of the constant $c$ is $-K_{00} K_{1 \overline{1}}$ and

$$
K_{01}(z, 0)=K_{11} z
$$

The last relation is equivalent to the relation (6.10), for $m=1, t=0$, hence, by Theorem $9, D$ is also a 1-representative domain with the origin as center.

CoROLlary. The relation (6.19) is a consequence of (7.3) for any domain which is conformally equivalent to the domain $D$ of Theorem 11. As there are domains for which (6.19) does not hold, e. g., a ring, for which (6.19) can be proved incorrect by a direct calculation (see [4] $p$. 10), one arrives at the conclusion that not all minimal domains are also representative domains with the same center, and that Theorem 5 does not hold if one replaces the circle by a general 1-minimal domain.

\section{REFERENCES}

1. S. Bergman, Ueber die Existenz von Repräsentantenbereichen, Math. Ann., 102 (1929), 430-446.

2. - Sur les fonctions orthogonales de plusieurs variables complexes avec les applications a la théorie des functions analytiques, Intersc. Pub., (1941) and Mém. des Sc. Math., 106, Paris (1947).

3. - Sur la fonction-noyan d'un domaine et ses applications dans la théorie des transformations pseudo-conformes, Mém. des Sc. Math., 108, Paris (1948).

4. - The lernel function and conformal mapping, Amer. Math. Soc., New-York (1950).

5. H. Kober, Dictionary of conformal representations, Dover Pub. (1952).

6. P. Kuffareff, Ueber das Zweifachzusammenhängende Minimalgebiet, Bull. Inst. Math. et Mec., Univ. de Tomsk, 1 (1935-1937), 228-236.

7. M. Maschler, Minimal domains and their Bergman kernel funciion, Pacific J. Math., 6 (1956), 501-516.

8. R. Nevanlinna, Uniformisierung, Springer-Verlag, Berlin, 1953 
9. M. Schiffer, Sur les domaines minima dans la théorie des transformations pseudo-conformes, C. R. Acad. Sci., Paris, 207 (1938), 112-115.

10. J. Tannery, J. Molk, Élémonts de la théorie des functions elliptiques, 1, Paris (1893).

11. K. I. Virtanen, Über Extremalfunktionen auf offenen Ricmannschen Flächen, Ann. Acad. Scient. Fenn. Ser. A. I. 141 (1952).

The Hebrew UnIVERSity, JeRUSALEM 


\section{PACIFIC JOURNAL OF MATHEMATICS}

\section{EDITORS}

\section{David Gilbarg}

Stanford University Stanford, California

\section{R. A. Beaumont}

University of Washington

Seattle 5, Washington

\section{A. L. Whiteman}

University of Southern California Los Angeles 7, California

L. J. Paige

University of California

Los Angeles 24, California

\section{ASSOCIATE EDITORS}

E. F. BECKENBACH
C. E. BURGESS
E. HEWITT
A. HORN

A. HORN

\author{
V. GANAPATHY IYER \\ R. D. JAMES \\ M. S. KNEBELMAN \\ L. NACHBIN
}

I. NIVEN

T. G. OSTROM

H. L. ROYDEN

M. M. SCHIFFER
E. G. STRAUS

G. SZEKERES

F. WOLF

K. YOSIDA

\section{SUPPORTING INSTITUTIONS}

\author{
UNIVERSITY OF BRITISH COLUMBIA \\ CALIFORNIA INSTITUTE OF TECHNOLOGY \\ UNIVERSITY OF CALIFORNIA \\ MONTANA STATE UNIVERSITY \\ UNIVERSITY OF NEVADA \\ OREGON STATE COLLEGE \\ UNIVERSITY OF OREGON \\ OSAKA UNIVERSITY \\ UNIVERSITY OF SOUTHERN CALIFORNIA
}

\author{
STANFORD UNIVERSITY \\ UNIVERSITY OF TOKYO \\ UNIVERSITY OF UTAH \\ WASHINGTON STATE COLLEGE \\ UNIVERSITY OF WASHINGTON \\ * * * * \\ AMERICAN MATHEMATICAL SOCIETY \\ CALIFORNIA RESEARCH CORPORATION \\ HUGHES AIRCRAFT COMPANY \\ SPACE TECHNOLOGY LABORATORIES
}

Mathematical papers intended for publication in the Pacific Journal of Mathematics should be typewritten (double spaced), and the author should keep a complete copy. Manuscripts may be sent to any one of the four editors. All other communications to the editors should be addressed to the managing editor, L. J. Paige at the University of California, Los Angeles 24, California.

50 reprints per author of each article are furnished free of charge; additional copies may be obtained at cost in multiples of 50 .

The Pacific Journal of Mathematics is published quarterly, in March, June, September, and December. The price per volume (4 numbers) is $\$ 12.00$; single issues, $\$ 3.50$. Back numbers are available. Special price to individual faculty members of supporting institutions and to individual members of the American Mathematical Society: $\$ 4.00$ per volume; single issues, $\$ 1.25$.

Subscriptions, orders for back numbers, and changes of address should be sent to Pacific Journal of Mathematics, 2120 Oxford Street, Berkeley 4, California.

Printed at Kokusai Bunken Insatsusha (International Academic Printing Co., Ltd.), No. 6, 2-chome, Fujimi-cho, Chiyoda-ku, Tokyo, Japan.

PUBLISHED BY PACIFIC IOURNAL OF MATHEMATICS, A NON-PROFIT CORPORATION

The Supporting Institutions listed above contribute to the cost of publication of this Journal, but they are not owners or publishers and have no responsibility for its content or policies. 


\section{Pacific Journal of Mathematics}

\section{Vol. 9, No. $3 \quad$ July, 1959}

Errett Albert Bishop, A minimal boundary for function algebras . . . . . . . . . . . . 629

John W. Brace, The topology of almost uniform convergence . . . . . . . . . . . . 643

Cecil Edmund Burgess, Chainable continua and indecomposability .......... 653

L. Carlitz, Multiplication formulas for products of Bernoulli and Euler

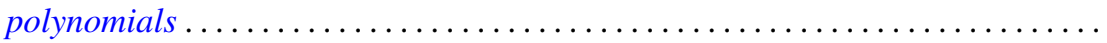

Eckford Cohen, A class of residue systems (mod $r$ ) and related arithmetical

functions. II. Higher dimensional analogues ....................

Shaul Foguel, Boolean algebras of projections of finite multiplicity . . . . . . . . . .

Richard Robinson Goldberg, Averages of Fourier coefficients .................

Seymour Goldberg, Ranges and inverses of perturbed linear operators .

Philip Hartman, On functions representable as a difference of convex functions ....

Milton Vernon Johns, Jr. and Ronald Pyke, On conditional expectation and

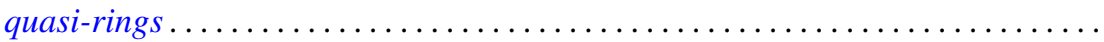

Robert Jacob Koch, Arcs in partially ordered spaces ....................

Gregers Louis Krabbe, A space of multipliers of type $L^{p}(-\infty, \infty) \ldots \ldots \ldots \ldots$

John W. Lamperti and Patrick Colonel Suppes, Chains of infinite order and their

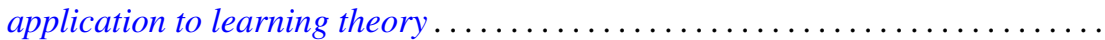

Edith Hirsch Luchins, On radicals and continuity of homomorphisms into Banach

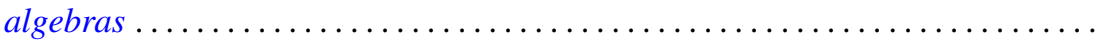

T. M. MacRobert, Multiplication formulae for the E-functions functions of their parameters.

Michael Bahir Maschler, Classes of minimal and representative domains and their kernel functions.

William Schumacher Massey, On the imbeddability of the real projective spaces in Euclidean space.

Thomas Wilson Mullikin, Semi-groups of class $\left(C_{0}\right)$ in $L_{p}$ determined by parabolic differential equations

Steven Orey, Recurrent Markov chains

Ernest Tilden Parker, On quadruply transitive groups ........ . .

Calvin R. Putnam, On Toeplitz matrices, absolute continuity, and unitary

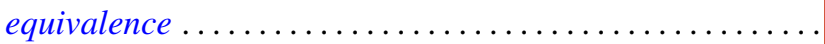

Helmut Heinrich Schaefer, On nonlinear positive operators.

Robert Seall and Marion Wetzel, Some connections between continued fractions and convex sets

Robert Steinberg, Variations on a theme of Chevalley

Olga Taussky and Hans Zassenhaus, On the similarity transformation between a

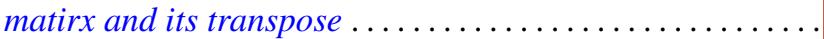

Emery Thomas, The suspension of the generalized Pontrjagin cohomology

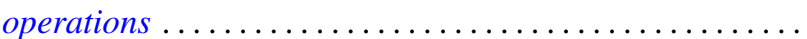

Joseph L. Ullman, On Tchebycheff polynomials ..................... 913

Richard Steven Varga, Orderings of the successive overrelaxation scheme ........ 925

Orlando Eugenio Villamayor, Sr., On weak dimension of algebras . 